\title{
Deontología y noticias falsas: estudio de las percepciones de periodistas españoles
}

\author{
Deontology and fake news: A study of the \\ perceptions of Spanish journalists
}

\author{
David Blanco-Herrero; Carlos Arcila-Calderón
}

Cómo citar este artículo:

Blanco-Herrero, David; Arcila-Calderón, Carlos (2019). “Deontología y noticias falsas: estudio de las percepciones de periodistas españoles". El profesional de la información, v. 28, n. 3, e280308.

https://doi.org/10.3145/epi.2019.may.08

Artículo recibido el 27-12-2018

Aceptación definitiva: 10-04-2019

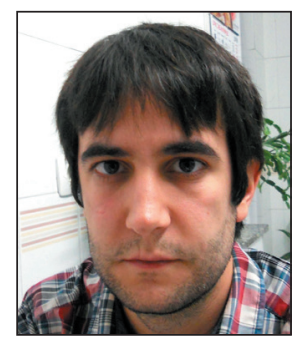

David Blanco-Herrero $\square$ https://orcid.org/0000-0002-7414-2998

Universidad de Salamanca

Campus Miguel de Unamuno, Edificio FES Avda. Francisco Tomás y Valiente, $\mathrm{s} / \mathrm{n}$. 37007 Salamanca, España david.blanco.herrero@usal.es

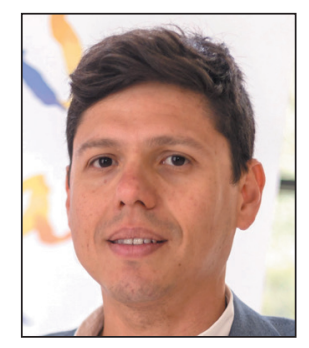

\section{Carlos Arcila-Calderón}

http://orcid.org/0000-0002-2636-2849

Universidad de Salamanca

Campus Miguel de Unamuno, Edificio FES

Avda. Francisco Tomás y Valiente, s/n.

37007 Salamanca, España

carcila@usal.es

\section{Resumen}

Las noticias falsas plantean uno de los principales desafíos del periodismo contemporáneo. El objetivo de esta investigación es conocer si la deontología periodística ofrece una herramienta útil para luchar contra las noticias falsas. Por medio de una encuesta a 383 periodistas españoles, así como cuatro entrevistas en profundidad a expertos del área, se comprobó la gravedad y el corte deontológico del problema de las noticias falsas, pero la ética periodística no fue considerada la medida más efectiva para luchar contra las fake news. También se buscaban posibles diferencias entre las opiniones de periodistas digitales y tradicionales en su aproximación a las noticias falsas y la deontología. Ambos grupos coinciden en responsabilizar del fenómeno a la situación económica del sector y la nueva forma de comunicación (no necesariamente periodística) en la Red, a lo que añaden la formación de los consumidores como una medida eficaz contra las noticias falsas.

\section{Palabras clave}

Noticias falsas; Fake news; Desinformación; Periodismo digital; Deontología periodística; Ética periodística; Periodistas; España.

\begin{abstract}
Fake news present one of the main challenges of contemporary journalism. The goal of this study is to know whether journalistic ethics offers a useful tool against fake news. A survey to 383 Spanish journalists, together with four in-depth interviews to experts in the field, showed the seriousness and the deontological dimension of the problem of fake news, but journalistic ethics was not considered to be the most useful strategy against fake information. We also wanted to look for possible differences in the approach of digital and traditional journalists towards fake news and deontology. Both groups agree in blaming the economic situation of the sector and the new online communication (not necessarily journalism) context for the rising of this phenomenon. Also, they point out the education of consumers as an effective action against fake news.
\end{abstract}

\section{Keywords}

Fake news; Disinformation; Digital journalism; Journalistic deontology; Journalistic ethics; Journalists; Spain. 


\section{Introducción}

Desde que en 2016 se asociaran con la victoria de Donald Trump o la decisión de los británicos de abandonar la Unión Europea a través del Brexit, las noticias falsas han pasado del debate en contextos académicos y en medios considerados de calidad a estar presentes en todos los ámbitos de la comunicación y la sociedad (McNair, 2017) y se han convertido en una de las mayores amenazas para la libertad de expresión, la democracia y el periodismo (Bennett; Livingston, 2018). Esto se produce en un contexto de posverdad, que el Oxford English Dictionary eligió en 2016 como palabra del año, definiéndola como

"las circunstancias en las que las emociones tienen un mayor peso que los hechos objetivos en la definición de la opinión pública"

y se asocia con el ascenso de opciones políticas populistas y radicales en un entorno comunicativo marcado por los medios sociales en la Red.

Esta asociación nos lleva a la creciente importancia de la comunicación online y las redes sociales, por lo que resulta esencial seguir ahondando en la posible relación entre periodismo digital y noticias falsas. Y dado que la deontología periodística apenas ha sido aplicada para ofrecer posibles soluciones a la materia en cuestión, el objetivo principal del artículo será observar si la deontología periodística es vista como una herramienta útil por los periodistas para enfrentarse a las noticias falsas. En términos más amplios, se busca ofrecer líneas de actuación que ayuden a disminuir la presencia e impacto de las fake news, incluyendo medidas menos relacionadas con la deontología.

De esta forma se quiere llenar una brecha de conocimiento existente en la bibliografía sobre el tema, a propósito de qué medidas pueden hacer frente a las noticias falsas, ampliando el estudio de esta materia todavía novedosa en la academia española, y abordando la problemática desde un campo menos habitual como la deontología. Metodológicamente, también se aspira seguir ampliando los trabajos que utilizan encuestas a profesionales y, sobre todo, estadística inferencial en áreas como la deontología periodística y las noticias falsas (Parra-Valero; Oliveira, 2018), en las que las técnicas cuantitativas son menos habituales.

En segundo término, para comprender en mayor profundidad el problema y sus soluciones, se compararán las opiniones de quienes trabajan en medios nativos digitales y quienes lo hacen en medios cuyo negocio original es tradicional, es decir, escrito, radiofónico, televisivo o de agencias. El objetivo es conocer si ambos tipos de periodistas presentan visiones distintas en torno a la importancia y las causas de las noticias falsas y en la utilidad que otorgan a la ética periodística en su aplicación en la lucha contra ellas. Esto permitirá seguir profundizando en las investigaciones que comparan las culturas profesionales y las opiniones de los periodistas tradicionales y los digitales.

\subsection{Noticias falsas y periodismo digital}

Para referirnos al fenómeno que vamos a investigar utilizaremos principalmente el término 'noticias falsas', junto a 'noticias falseadas', recomendado por la Fundéu, y el anglosajón 'fake news', por su gran popularidad. Debemos puntualizar que Bennett y Livingston (Fundéu, 2018, p. 124) distinguen el término 'fake news', centrado en "incidentes aislados de confusión y mentira", de la desinformación, que sería "la irrupción sistemática de flujos de información autoritarios". Así, este trabajo se centra en los casos, intencionados o no, que el estudio de expertos encargado por la Comisión Europea considera 'fake news', más que con el más amplio y complejo fenómeno de la desinformación (Comisión Europea, 2018). No obstante, las noticias falsas deben entenderse como un elemento íntimamente relacionado tanto con la citada desinformación, que es

El fenómeno de las noticias falseadas en la actualidad, derivado de la ecología de los medios digitales, no puede entenderse aislado de la desinformación y la posverdad

la problemática mayor dentro de la que se incluyen las informaciones falseadas, como con la posverdad, en la que las emociones ganan peso en el discurso público en detrimento de los hechos. A pesar de lo anterior, todavía no existe consenso para definir con precisión los términos que rodean a las noticias falsas y la desinformación (Martens et al., 2018; Tandoc Jr.; Lim; Ling, 2017), por lo que conviene anotar los elementos que caracterizan a las fake news a las que nos enfrentamos en la actualidad.

Éstas vienen definidas por la relevancia que alcanzan gracias a su amplia difusión a través de los medios online y, más concretamente, las redes sociales (Allcott; Gentzkow, 2017). También es característico el uso de lo que McStay (2016) denomina "empathic media", es decir, una serie de tecnologías de la comunicación capaces de evaluar y almacenar sentimientos humanos, incluyendo el análisis de sentimientos en redes sociales, y utilizarlo con el fin de manipular la opinión pública. Según Bakir y McStay (2018) las noticias falsas han alcanzado su dimensión actual gracias a la ecología de los medios digitales, explicada por:

- decadencia financiera de los medios de comunicación tradicionales;

- inmediatez impuesta por el entorno digital;

- creación y rápida circulación de desinformación, generada con fines espurios o por ignorancia;

- creciente 'emocionalización' del discurso;

- beneficio que generan los algoritmos utilizados por los medios sociales. 
Waisbord apunta en esta línea hacia el caos contemporáneo de la comunicación pública, provocado en buena medida por la

"transformación de la esfera pública motivada por la proliferación digital de oportunidades para la expresión pública y el encuentro de comunidades con diferentes aproximaciones a las noticias y la información" (Waisbord, 2018, p. 1869).

A esto cabe añadir el hecho de que los rumores y noticias falseadas se puedan llegar a extender con mayor rapidez que las verdaderas (Vosoughi; Roy; Aral, 2018). Finalmente, de acuerdo con Tandoc Jr., Lim y Ling (2017), el consumidor también juega un papel relevante en la construcción de este fenómeno, pues la no credibilidad de una noticia falseada o la falta de efecto de la misma eliminarían uno de los elementos clave de su existencia.

El presente trabajo se enmarcará dentro del campo de los estudios sobre periodismo, una línea que también ha sido adoptada con frecuencia al estudiar las fake news (Bakir; McStay, 2018; Marchi, 2012; Tandoc Jr.; Lim; Ling, 2017; Alonso, 2017). En España, aunque la investigación sobre noticias falsas, posverdad o desinformación tiene menor recorrido, ha ido ganando fuerza en los últimos años (Marcos-Recio, 2017; Marcos-Recio; Sánchez-Vigil; Olivera-Zaldua, 2017; Elías, 2018; Parra-Valero; Oliveira, 2018).

Por su parte, en los trabajos que han observado cómo el periodismo online ha afectado al escenario informativo, ha sido frecuente que las opiniones sobre las ventajas e inconvenientes del periodismo digital muestren una gran disparidad (Suárez-Villegas, 2015; Micó-Sanz et al., 2008). Así, la inmediatez o la democratización de la información, fortalezas del periodismo en la Red y, sobre todo, de las redes sociales (Carlson, 2018), se pueden convertir en importantes riesgos si hacen disminuir la calidad y rigurosidad de los contenidos (Suárez-Villegas; Cruz-Álvarez, 2016). De hecho, es en este tipo de plataformas donde los investigadores sobre noticias falsas han puesto el foco con mayor frecuencia (Allcott; Gentzkow, 2017; Grinberg et al., 2019; Martens et al., 2018; Vosoughi; Roy; Aral, 2018)

Otra de las observaciones más habituales es la creciente digitalización del mercado informativo (Marcos-Recio; García-Alonso; Parra-Valcarce, 2014) que, unido a la crisis económica, la caída en las ventas de diarios o el descenso de la publicidad ha dado lugar a una industria más débil y precaria (Figueras-Maz et al., 2012), lo que a su vez ha facilitado la aparición de las noticias falsas (Bakir; McStay, 2018). Vemos por tanto una vinculación entre periodismo y comunicación digital y noticias falsas. Con esto, el estudio se pregunta:

(PI1) ¿cuáles son las causas y el grado de importancia de las noticias falsas para los periodistas españoles?

Y dado el gran interés en seguir estudiando las culturas profesionales de los periodistas (Rodríguez-Martínez et al., 2017; Suárez-Villegas et al., 2017) y las diferencias entre los periodistas de medios nativos digitales y aquellos de medios que se pueden considerar tradicionales (Berganza; Arcila-Calderón; Herrero-Jiménez, 2016; Gómez-Mompart; Gutiérrez-Lozano; Palau-Sampio, 2015; Alonso-González, 2016), se investigará también:

(PI1.1) ¿existen diferencias entre periodistas digitales y tradicionales con respecto a esta materia?

\subsection{Deontología periodística}

Abundan las investigaciones sobre los desafíos éticos que plantean las redes sociales (Santín, 2017; Suárez-Villegas, 2017; Colussi-Ribeiro, 2016) o el periodismo digital en general (Hayes; Singer; Ceppos, 2007). Sin embargo, Micó-Sanz et al. (2008), Parra-Valcarce, Real-Rodríguez y López-Talavera (2017), y Díaz-Campo y Segado-Boj (2014; 2015) señalan la necesidad de la deontología y sus normativas de adaptarse al contexto de la convergencia digital, mientras Blanco-Herrero (2018) añade la nula atención que dichas normas prestan a las noticias falseadas. De hecho, puede resultar llamativo que, aunque parece existir una relación entre las noticias falsas y la deontología (Clark; Zhou, 2015), ésta no es una de las perspectivas más utilizadas para analizar este fenómeno. Resulta interesante descubrir si:

(PI2) ¿ perciben los periodistas españoles la deontología periodística como una herramienta útil en la lucha contra las fake news?

Al mismo tiempo, las opiniones de periodistas digitales y de medios tradicionales han despertado un particular interés (Suárez-Villegas, 2015; Tirado-Pascual, 2016; Diezhandino-Nieto, 2008), destacando el mayor peso que los periodistas vinculados a medios tradicionales otorgan a algunos principios éticos, pero la bibliografía es todavía escasa en este aspecto. Y dado que la metodología de encuesta a profesionales ha sido frecuente en los estudios del sector (Figueras-Maz et al., 2012; Humanes, 1998; Clark; Zhou, 2015), se pretende descubrir:

(PI2.1) ¿son los periodistas de medios nativos digitales o de medios tradicionales los que mayor utilidad ven en esta vía?

Parra-Valero y Oliveira (2018) recogieron las medidas propuestas por la investigación académica para hacer frente a las noticias falsas, destacando las medidas de fact-checking, los procesos colaborativos en los que los ciudadanos evalúan las noticias, la alfabetización informacional y los procesos computacionales automatizados. Así, con la intención de complementar estas líneas de investigación y poner las medidas deontológicas en contexto, también se busca conocer:

(PI3) ¿qué tipo de actuaciones pueden resultar más efectivas para evitar la propagación de noticias falsas desde los medios de comunicación?

(PI3.1) ¿ existen diferencias entre periodistas digitales y tradicionales en estas percepciones? 


\section{Metodología}

La investigación está compuesta por una encuesta a profesionales y por una serie de entrevistas en profundidad a expertos en la materia. De esta forma, se sigue la línea de estudios como el de Maciá-Barber y Herrera-Damas (2009) o el de Luengo, Maciá-Barber y Requejo-Alemán (2017), combinando una técnica cuantitativa, para analizar estadísticamente las percepciones de los periodistas españoles, y una cualitativa, para profundizar en los argumentos y opiniones de los periodistas desde la óptica de cuatro expertos del área.

\subsection{Encuesta a profesionales}

\subsubsection{Muestra y procedimiento}

El cuestionario se redactó a partir de la revisión del contenido de dieciséis códigos deontológicos periodísticos (siguiendo a Blanco-Herrero, 2018) y de la consulta de piezas periodísticas y académicas sobre la materia (Bakir; McStay, 2018; Berganza; Arcila-Calderón; Herrero-Jiménez, 2016; Díaz-Campo; Segado-Boj, 2015; Marchi, 2012; Marcos-Recio; Sánchez-Vigil; Olivera-Zaldua, 2017; Micó-Sanz et al., 2008; Parra-Valcarce; Real-Rodríguez; López-Talavera, 2017; Suárez-Villegas, 2015; Tandoc Jr.; Lim; Ling, 2017), completándose con los comentarios y evaluaciones recibidos durante la fase de validación. Para el diseño del instrumento se utilizó la plataforma online Qualtrics, a través de la cual también se distribuyó. Las pruebas estadísticas se realizaron con el programa SPSS (versión 20).

De esta forma, la primera etapa en la construcción del cuestionario fue la operacionalización de las variables, para lo que se recurrió a dos expertos independientes del sector, que pudieron evaluar si los 50 items generados y las variables preliminares respondían a los objetivos del estudio. Posteriormente, teniendo en cuenta sus consideraciones, se realizó una prueba piloto de test-retest entre los días 12 de abril y 4 de mayo de 2018 para medir la fiabilidad y la estabilidad del instrumento. Se contactó con 60 miembros de la muestra para que respondieran al cuestionario en dos ocasiones con unos doce días de diferencia y así poder comparar la estabilidad en el tiempo de las respuestas. De los 54 items que contenía este cuestionario, se eliminó uno y se modificó la redacción de siete, pues se encontraban por debajo de 0,45 en la prueba del coeficiente de correlación intraclase. El coeficiente medio fue de 0,69 que, para un estudio exploratorio como éste, puede considerarse adecuado (Kaplan; Saccuzzo, 1982). Hechas estas modificaciones, se comenzó a distribuir el cuestionario definitivo el 8 de mayo. Esta última versión contaba con 53 items, de los que diez no serían incluidos en el análisis por no resultar significativos en los análisis factoriales exploratorios (ver apartado siguiente).

Se consideró como universo al conjunto de periodistas españoles, independientemente de sus condiciones sociodemográficas o del tipo de periodismo que realizaran. Para ello se contactó a todas las asociaciones de la prensa federadas o vinculadas a la FAPE (Federación de Asociaciones de Periodistas de España), así como a la redacción de los medios españoles analizados en el EGM (Estudio general de medios) o por la Oficina de Justificación de la Difusión (OJD), para que distribuyeran el cuestionario entre sus trabajadores o asociados. También se utilizaron las redes sociales Facebook y Twitter para aumentar el alcance de la encuesta. Salvo en ocasiones puntuales, la mayoría de solicitudes se realizó a través de correo electrónico. En todos estos contactos, en especial cuando se trataba de asociaciones profesionales, se solicitaba ayuda en la difusión del cuestionario entre colegas o a través de sus redes sociales, siguiendo una distribución no probabilística de tipo bola de nieve. La recolección de respuestas se cerró en agosto de 2018. Antes de comenzar el estudio de los resultados se llevó a cabo un análisis exploratorio de los datos (AED) para controlar que no existían inconsistencias y para evitar respuestas repetidas o incompletas.

Debido a que el estudio buscaba comparar medios tradicionales con medios nativos digitales, se incidió de manera especial en estos últimos, contactando también a los medios de comunicación digitales creados por periodistas entre 2008 y 2015 (Asociación de la Prensa de Madrid, 2015), para contar de esta manera con una mayor presencia de este tipo de periodistas. Como ocurre en la investigación de Suárez-Villegas (2015), se entiende que esta estrategia no perjudica necesariamente la representatividad de la muestra, pues no se busca conocer la cantidad de periodistas de cada tipo, sino las diferentes aproximaciones de éstos a la deontología y a las noticias falsas, por lo que se trataría de comparar los datos de dos submuestras independientemente del peso de cada una de ellas en la muestra conjunta. Por lo tanto, si bien los datos no pueden ser generalizables debido al sesgo de auto-exposición, la alta cantidad de respuestas recibidas de los medios y asociaciones contactadas da cuenta de una muestra con características cercanas a la de la población de periodistas del país.

El número de respuestas fue de 383, muy cercano a la muestra que se ha utilizado en trabajos como los de Berganza, Arcila-Calderón y Herrero-Jiménez (2016) o Sánchez-Aranda y Rodríguez-Andrés (1999), con 390 y 392 respuestas respectivamente. Los primeros en 2014 estimaban alrededor de 18.000 periodistas en España, mientras que la FAPE cuenta con unos 19.000 asociados, por lo que, utilizando este último valor, podríamos hablar de un tamaño de muestra adecuado con $\mathrm{N}=19.000$, un $95 \%$ de confianza y un error del $5 \%$.

La muestra estaba formada por un $60,1 \%$ de hombres, un $38,6 \%$ de mujeres y un $1,3 \%$ de personas que no indicaron su género; con una edad media de 43,2 años (DT=11,91). 252 de los periodistas encuestados (un 66,1\%) tienen un empleo a tiempo completo, frente a los 25 (el 6,6\%) que trabajan a tiempo parcial y a los 70 periodistas freelance (el $18,4 \%$ ). A esto se suman 18 desempleados, que representan el $4,7 \%$ de los encuestados, y un grupo de otras 16 personas, un $4,2 \%$, que agrupa a jubilados, becarios, trabajadores no remunerados o pluriempleados. 
En cuanto al tipo de periodismo que realizan los encuestados, 127 de ellos (el 33,2\%) forman parte de medios escritos (sean diarios, revistas o suplementos). También hay 37 periodistas televisivos (el 9,7\%), 40 que trabajan en radio (el $10,4 \%$ ) y 24 en agencias (el 6,3\%). Esto hace un total de 228 periodistas tradicionales, alcanzando el 59,5\% de la muestra. Entre los periodistas digitales, 143 de ellos (el 37,3\% del total), trabajan en diarios online o blogs, mientras los 12 restantes (el 3,1\%) lo hacen en plataformas como YouTube, redes sociales, etc. El total de periodistas digitales asciende al $40,5 \%$ de la muestra.

\subsubsection{Medidas}

La parte central del cuestionario estaba formada por preguntas cerradas con una estructura de escala tipo Likert de cinco puntos con diferentes afirmaciones, que se agruparon luego en constructos para dar respuesta a las variables estudiadas. Se realizaron tres análisis factoriales exploratorios (AFE) para medir la validez de los constructos (Igartua-Perosanz, 2006; Berganza; Arcila-Calderón; Herrero-Jiménez, 2016). Tras esto se procedió a eliminar los items con cargas más bajas o duplicadas.

En el primer análisis -con las dimensiones relacionadas con la deontología en términos generales- los items presentan una carga significativa en alguno de los dos factores sugeridos como estructura latente (varianza explicada $=65,25 \%$, $\mathrm{KMO}=0,849$, test de esfericidad de Bartlett: $\left.\left[\mathrm{X}^{2}(28)=1204,75, \mathrm{p}<0,001\right]\right)$.

En el segundo AFE - con las dimensiones relacionadas con las causas de las noticias falsas- los items poseen una carga significativa en uno de los cinco factores sugeridos como estructura latente (varianza explicada $=68,95 \%, \mathrm{KMO}=0,826$, test de esfericidad de Bartlett: $\left.\left[X^{2}(136)=2693,92, p<0,001\right]\right)$. Estos constructos midieron las causas que los periodistas perciben como importantes en la proliferación de las noticias falsas.

Finalmente, en el tercer AFE -con las dimensiones relacionadas con las medidas que deben tomarse contra las noticias falsas- los items arrojaron cargas significativas en alguno de los cuatro factores sugeridos (varianza explicada $=74,39 \%$, $\mathrm{KMO}=0,794$, test de esfericidad de Bartlett: $\left.\left[\mathrm{X}^{2}(66)=2170,43, \mathrm{p}<0,001\right]\right)$. Todos los alpha de Cronbach $(\alpha)$ mostraron valores adecuados, siempre por encima de 0,69 (Cronbach, 1951).

Con esto se obtuvieron once constructos. Todos ellos están medidos del 1 al 5, una mayor puntuación implica una mayor valoración de la medida, sea esta importancia, efectividad, relación o gravedad. Estas dimensiones son:

- Gravedad de las fake news para el periodismo: con cinco items $(\alpha=0,843)$.

- Relación entre noticias falsas y deontología periodística: incluye tres items $(\alpha=0,769)$.

- Responsabilidad de los problemas propios del periodismo en la proliferación de las noticias falsas: formado por cinco items $(\alpha=0,842)$, entiende el periodismo de forma global.

- Responsabilidad de internet y las nuevas formas de comunicar en la proliferación de las noticias falsas: formado por cinco items $(\alpha=0,774)$.

- Responsabilidad de aspectos económicos en la proliferación de las noticias falsas: formada por tres items $(\alpha=0,840)$, alude a factores asociados a la situación económica.

- Responsabilidad del nuevo contexto internacional en la proliferación de las noticias falsas: con dos items $(\alpha=0,686)$.

- Responsabilidad de la incorrecta adaptación de los medios tradicionales a la nueva realidad en la proliferación de las noticias falsas: compuesta por dos items $(\alpha=0,853)$.

- Efectividad de medidas formativas entre los periodistas para luchar contra las noticias falsas: formado por cuatro items $(\alpha=0,844)$, incluye también aquellas relacionadas con la cultura profesional periodística.

- Efectividad de medidas obligatorias para luchar contra las noticias falsas: compuesta por cuatro items $(\alpha=0,805)$, hacía alusión a medidas que llevan asociadas sanciones por incumplimiento, incluyendo la legislación.

- Efectividad de medidas formativas entre los consumidores para luchar contra las noticias falsas: formado por dos items $(\alpha=0,810)$.

- Efectividad de medidas no obligatorias para luchar contra las noticias falsas: formada por dos items $(\alpha=0,886)$, se refiere a las normas voluntarias o autoimpuestas.

Salvo la cuestión referida al tipo de periodismo realizado, no se forzó ninguna de las respuestas, pues se consideraba que la posible ignorancia o falta de opinión sobre determinados asuntos podría dar lugar a opiniones sesgadas o artificiales.

\subsubsection{Análisis}

Las respuestas fueron anonimizadas y trasladadas al programa SPSS y, de acuerdo con las variables confirmadas por cada AFE, se crearon los constructos a través del promedio de los items correspondientes. Para controlar que no existían inconsistencias se llevó a cabo un análisis exploratorio de los datos (AED) y se comprobó la distribución gráfica de las frecuencias. La parte central del análisis estuvo compuesta por las pruebas bivariadas de diferencia de medias t de Student para muestras independientes. También se llevaron a cabo correlaciones con vocación exploratoria y pruebas chi cuadrado para comparar si determinadas variables nominales influían sobre otras. El error tipo I se determinó en el $95 \%(\alpha=0,05)$. 


\subsection{Entrevistas en profundidad}

Entre marzo y abril de 2019, una vez cerrado el análisis cuantitativo, se llevaron a cabo entrevistas en profundidad con expertos en periodismo digital y en el área de las noticias falsas que pudieran complementar y valorar los resultados de la encuesta, indagando en ellos y en su interpretación con más detalle. Se realizaron cuatro entrevistas semi-estructuradas con preguntas abiertas; tres fueron telefónicas, de entre 10 y 15 minutos de duración, y la cuarta se realizó por correo electrónico. Se pidió a los expertos que valoraran las observaciones realizadas durante la etapa de encuesta, ampliando sus respuestas con causas de la aparición del fenómeno o con posibles medidas que tal vez no se hubieran contemplado en la encuesta. Siguiendo el esquema de la encuesta y del resto de la investigación, las preguntas se dividieron en tres bloques:

- noticias falsas: gravedad y causas;

- deontología: relación con las noticias falsas y utilidad para enfrentarlas);

- medidas para luchar contra las noticias falsas.

Los expertos consultados fueron:

- J. Z.: periodista y profesor universitario, experto en periodismo digital.

- C. R.: periodista y responsable de nuevas narrativas en uno de los principales diarios españoles.

- J. G.: periodista, investigador, subdirector de uno de los matinales de más audiencia de la televisión nacional y profesor universitario.

- L. B.: periodista y una de las principales responsables de una de las iniciativas de fact-checking más conocidas en España.

\section{Resultados}

En términos generales, se observa que los periodistas digitales son significativamente más jóvenes ( $n=155 ; M=40,95$; $\mathrm{DT}=11,98)$ que los de medios tradicionales $(\mathrm{n}=219 ; \mathrm{M}=44,74 ; \mathrm{DT}=11,62), \mathrm{t}(372)=2,067, \mathrm{p}<0,01, \mathrm{~d}=0,21$. También se comprobó que, aunque el periodismo digital tiene una presencia proporcional ligeramente superior de mujeres, las diferencias no son significativas $\left[\chi^{2}(1,378)=0,079, p>0,05\right]$.

\subsection{Causas e importancia de las noticias falsas}

Respondiendo a la PI1, las noticias falsas se perciben (tabla 1) como algo bastante grave $(M=4,18)$. Las causas (tabla 2) que suelen considerarse más relevantes en la proliferación de este fenómeno son las nuevas formas de comunicar en la Red $(M=3,88)$, seguidas de los aspectos económicos $(M=3,77)$ y de las derivadas del sec-

La nueva forma de comunicación online y los aspectos económicos se perciben como principales culpables de la proliferación de las noticias falsas tor del periodismo en su conjunto $(\mathrm{M}=3,65)$. Tanto el proceso de actualización de los medios tradicionales al nuevo paradigma informativo $(M=3,42)$ como el cambiante contexto global $(M=2,89)$ son considerados menos relevantes. Entrando en items más concretos, tanto las redes sociales $(M=4,47)$ como la precariedad $(M=4,08)$ son considerados elementos de mucho peso en la aparición de las noticias falsas.

Tabla 1. Valores de la dimensión: Gravedad de las fake news para el periodismo

\begin{tabular}{|c|c|c|c|c|c|c|}
\hline & M & DT & $\mathbf{M}_{\text {trad }}$ & DT $_{\text {trad }}$ & $\mathbf{M}_{\mathrm{dig}}$ & DT $_{\text {dig }}$ \\
\hline Gravedad de las fake news para el periodismo & 4,18 & 0,81 & 4,24 & 0,79 & 4,09 & 0,83 \\
\hline $\begin{array}{l}\text { Consideración de las noticias falsas como uno de los fenómenos que más dañan al perio- } \\
\text { dismo* }\end{array}$ & 4,46 & 0,99 & 4,55 & 0,91 & 4,33 & 1,10 \\
\hline Nivel de prioridad que debería tener para el periodismo acabar con las noticias falsas & 4,44 & 0,93 & 4,46 & 0,94 & 4,41 & 0,93 \\
\hline Importancia de las noticias falsas en eventos de gran calado de la sociedad & 4,27 & 0,92 & 4,33 & 0,90 & 4,18 & 0,94 \\
\hline Pérdida de credibilidad del sector periodístico a raíz del fenómeno de las noticias falsas* & 3,98 & 1,11 & 4,07 & 1,03 & 3,84 & 1,21 \\
\hline Necesidad de erradicar las noticias falsas para la supervivencia de la profesión periodística & 3,74 & 1,18 & 3,77 & 1,19 & 3,70 & 1.18 \\
\hline
\end{tabular}

$*$ = diferencias entre periodistas tradicionales y digitales significativas al $95 \%$

(M: media; DT: desviación típica) 
Tabla 2. Valores de las dimensiones relacionadas con las causas de la proliferación de las noticias falsas

\begin{tabular}{|c|c|c|c|c|c|c|}
\hline $\begin{array}{l}\text { Responsabilidad de los problemas propios del periodismo en la proliferación de las noti- } \\
\text { cias falsas }\end{array}$ & 3,65 & 0,89 & 3,67 & 0,91 & 3,62 & 0,85 \\
\hline Concentración de los grandes medios en pocas manos & 3,60 & 1,17 & 3,62 & 1,21 & 3,57 & 1,13 \\
\hline Ideologización de los medios de comunicación & 3,78 & 1,16 & 3,81 & 1,16 & 3,75 & 1,17 \\
\hline Falta de independencia de los medios de comunicación & 3,95 & 1,10 & 3,95 & 1,12 & 3,95 & 1,07 \\
\hline Conversión de las empresas informativas en conglomerados con múltiples ramas de negocio & 3,69 & 1,13 & 3,75 & 1,08 & 3,60 & 1,20 \\
\hline Los propios profesionales & 3,24 & 1,09 & 3,23 & 1,13 & 3,26 & 1,04 \\
\hline $\begin{array}{l}\text { Responsabilidad de internet y las nuevas formas de comunicar en la proliferación de las } \\
\text { noticias falsas* }\end{array}$ & 3,88 & 0,74 & 3,95 & 0,75 & 3,78 & 0,73 \\
\hline Aparición de nuevos medios de comunicación digitales & 3,50 & 1,09 & 3,58 & 1,11 & 3,39 & 1,06 \\
\hline Las redes sociales & 4,47 & 0,82 & 4,49 & 0,82 & 4,44 & 0,83 \\
\hline Los buscadores online* & 3,69 & 1,06 & 3,79 & 1,06 & 3,54 & 1,06 \\
\hline Proliferación de fenómenos comunicativos no convencionales & 3,97 & 1,05 & 4,05 & 1,04 & 3,86 & 1,05 \\
\hline Pérdida de influencia de los medios de comunicación tradicionales & 3,77 & 1,09 & 3,84 & 1,07 & 3,66 & 1,11 \\
\hline Responsabilidad de aspectos económicos en la proliferación de las noticias falsas & 3,77 & 0,96 & 3,77 & 0,94 & 3,78 & 0,98 \\
\hline Precarización del empleo de los periodistas & 4,08 & 1,07 & 4,08 & 1,07 & 4,08 & 1,09 \\
\hline Crisis en el sector & 3,88 & 1,09 & 3,88 & 1,06 & 3,88 & 1,13 \\
\hline Crisis económica en general & 3,36 & 1,13 & 3,35 & 1,12 & 3,38 & 1,16 \\
\hline Responsabilidad del nuevo contexto internacional en la proliferación de las noticias falsas & 2,89 & 0,99 & 2,93 & 1,03 & 2,84 & 0,93 \\
\hline La globalización & 3,04 & 1,15 & 3,04 & 1,18 & 3,03 & 1,10 \\
\hline Aparición de nuevas materias de las que informar & 2,74 & 1,12 & 2,81 & 1,15 & 2,65 & 1,07 \\
\hline $\begin{array}{l}\text { Responsabilidad de la incorrecta adaptación de los medios tradicionales a la nueva reali- } \\
\text { dad en la proliferación de las noticias falsas }\end{array}$ & 3,42 & 1,05 & 3,37 & 1,04 & 3,49 & 1,08 \\
\hline Falta de actualización de los medios tradicionales al nuevo escenario comunicativo & 3,49 & 1,09 & 3,42 & 1,09 & 3,58 & 1,07 \\
\hline Incorrecta incorporación de los medios tradicionales a lo digital & 3,34 & 1,17 & 3,30 & 1,13 & 3,40 & 1,21 \\
\hline
\end{tabular}

$*$ diferencias entre periodistas tradicionales y digitales significativas al $95 \%$

(M: media; DT: desviación típica)

Desde una visión comparada, para responder a la PI1.1, no se aprecian diferencias significativas entre periodistas digitales y tradicionales al valorar la gravedad ni las causas de la aparición de las noticias falsas, por lo que cabría afirmar que la concepción del fenómeno es similar en ambos grupos. Sí se observa (tabla 2), no obstante, que la responsabilidad atribuida a internet y las nuevas formas de comunicar es menor entre los periodistas digitales $(M=3,78 ; D T=0,73)$ que entre los tradicionales $(M=3,95 ; D T=0,75), t(381)=2,204, p<0,05, d=0,23$.

\subsection{Utilidad de la deontología para luchar contra las fake news}

La relación existente entre deontología periodística y la problemática de las noticias falsas (tabla 3) parece incuestionable ( $M=4,28)$. No obstante, las medidas voluntarias aplicadas a los periodistas (tabla 4), en las que se basa la deontología como norma autoimpuesta, son las consideradas menos efectivas $(M=2,86)$. Les siguen las medidas obligatorias, dentro de las que también podrían englobarse normativas deontológicas que lleven aparejadas sanciones por incumplimiento $(M=3,38)$. La formación de los periodistas, que también guarda una cierta aunque distante, relación con la deontología, se considera bastante efectiva $(M=3,98)$, mientras que las actuaciones aplicables a los consumidores, con las que menor conexión tiene la deontología periodística, son las que se consideran más efectivas ( $M=4,17)$.

Tabla 3. Valores de la dimensión: Relación entre noticias falsas y deontología periodística

Relación entre noticias falsas y deontología periodística

Consideración de las noticias falsas como un problema de componente ético

Posibilidad de que mayores estándares éticos hubieran impedido que las noticias falsas alcanzaran la dimensión que han logrado

Consideración de la deontología periodística como una herramienta esencial para enfrentarse a las noticias falsas

\begin{tabular}{|c|c|c|c|c|c|}
\hline $\mathbf{M}$ & $\mathbf{D T}$ & $\mathbf{M}_{\text {trad }}$ & $\mathbf{D T}_{\text {trad }}$ & $\mathbf{M}_{\text {dig }}$ & $\mathbf{D T}_{\text {dig }}$ \\
\hline 4,28 & 0,82 & 4,26 & 0,85 & 4,31 & 0,78 \\
\hline 4,52 & 0,85 & 4,51 & 0,90 & 4,54 & 0,78 \\
\hline 3,97 & 1,16 & 3,94 & 1,16 & 4,01 & 1,17 \\
\hline 4,35 & 0,94 & 4,34 & 0,96 & 4,36 & 0,90 \\
\hline
\end{tabular}

(M: media; DT: desviación típica) 
Tabla 4. Valores de las dimensiones relacionadas con las medidas para hacer frente a las noticias falsas

\begin{tabular}{|c|c|c|c|c|c|c|}
\hline & M & DT & $M_{\text {trad }}$ & $\mathrm{DT}_{\text {trad }}$ & $M_{\text {dig }}$ & $\mathrm{DT}_{\text {dig }}$ \\
\hline $\begin{array}{l}\text { Efectividad de medidas formativas entre los periodistas para luchar contra las noti- } \\
\text { cias falsas }\end{array}$ & 3,98 & 0,87 & 3,97 & 0,83 & 3,99 & 0,93 \\
\hline Efectividad de desarrollar una cultura profesional que rechace prácticas irresponsables & 4,28 & 0,97 & 4,25 & 0,96 & 4,32 & 0,99 \\
\hline Efectividad de la formación universitaria & 4,00 & 1,08 & 4,02 & 1,03 & 3,96 & 1,16 \\
\hline Efectividad de la formación durante la etapa laboral & 4,02 & 1,05 & 4,04 & 0,98 & 3,99 & 1,15 \\
\hline Efectividad de campañas de concienciación dirigidas a los periodistas & 3,62 & 1,12 & 3,58 & 1,10 & 3,67 & 1,16 \\
\hline Efectividad de medidas obligatorias para luchar contra las noticias falsas & 3,38 & 0,90 & 3,37 & 0,90 & 3,39 & 0,92 \\
\hline Efectividad de la legislación regional, nacional o supranacional & 3,32 & 1,13 & 3,31 & 1,16 & 3,34 & 1,09 \\
\hline Efectividad de las normativas vinculantes del propio medio & 3,69 & 1,03 & 3,70 & 1,04 & 3,67 & 1,02 \\
\hline Efectividad de las normativas vinculantes de otras instituciones & 3,34 & 1,13 & 3,35 & 1,11 & 3,34 & 1,16 \\
\hline Efectividad de controlar a los medios de comunicación como instituciones & 3,15 & 1,26 & 3,11 & 1,30 & 3,21 & 1,19 \\
\hline $\begin{array}{l}\text { Efectividad de medidas formativas entre los consumidores para luchar contra las } \\
\text { noticias falsas** }\end{array}$ & 4,17 & 0,95 & 4,05 & 0,99 & 4,36 & 0,85 \\
\hline Efectividad de campañas de concienciación dirigidas a los consumidores ${ }^{* *}$ & 4,04 & 1,08 & 3,89 & 1,12 & 4,25 & 0,98 \\
\hline $\begin{array}{l}\text { Efectividad de desarrollar entre los consumidores una cultura que rechace prácticas perio- } \\
\text { dísticas irresponsables** }\end{array}$ & 4,30 & 0,99 & 4,20 & 1,05 & 4,46 & 0,87 \\
\hline Efectividad de medidas no obligatorias para luchar contra las noticias falsas & 2,86 & 1,10 & 2,86 & 1,08 & 2,85 & 1,13 \\
\hline Efectividad de normativas no vinculantes del propio medio & 2,92 & 1,15 & 2,92 & 1,14 & 2,94 & 1,16 \\
\hline Efectividad de normativas no vinculantes de otras asociaciones & 2,79 & 1,17 & 2,81 & 1,12 & 2,75 & 1,24 \\
\hline
\end{tabular}

$* *$ diferencias entre periodistas tradicionales y digitales significativas al $99 \%$

(M: media; DT: desviación típica)

Para responder a la PI2, podemos afirmar que la deontología periodística, a pesar de su notable conexión con las noticias falsas, no es percibida como una herramienta de gran utilidad para enfrentarlas.

Las medidas asociadas a los consumidores (tabla 4) fueron las únicas que mostraron diferencias significativas, $\mathrm{t}(381)=$ $-3,20, p<0,01, d=0,33$, pues los periodistas digitales las consideran más efectivas $(M=4,36$; DT $=0,85)$ que los periodistas que trabajan en otros medios $(M=4,05 ; D T=1,00)$. Por lo tanto se puede responder a la PI2.1 que los periodistas digitales y los tradicionales no muestran diferencias significativas en la utilidad que asignan a la deontología como medida en la lucha contra las noticias falseadas.

\subsection{Medidas para luchar contra las noticias falsas}

Como se ha indicado (tabla 4), los periodistas consideran que las medidas más efectivas para enfrentarse a las fake news desde el sector periodístico son aquellas relacionadas con el desarrollo de medidas de alfabetización orientadas a los consumidores, seguidas de las medidas formativas entre los periodistas, mientras que se consideran menos útiles las medidas obligatorias, vinculantes y sancionadoras y, especialmente, la regulación no obligatoria.

Para responder a la PI3 de forma más detallada y con medidas más concretas podemos desgranar los items de las cuatro dimensiones referidas a las medidas que se pueden llevar a cabo para luchar contra las noticias falsas (tabla 4). Así observamos que el tipo de actuación considerado más efectivo es el desarrollo de una cultura que rechace prácticas irresponsables -en las que se incluirían las noticias falsas-, tanto entre los consumidores $(M=4,30)$ como entre los propios periodistas $(M=4,28)$. Las campañas de concienciación dirigidas a los consumidores $(M=4,04)$, formación durante la etapa laboral de los profesionales $(M=4,02)$ y una mayor formación universitaria de estos $(M=4,00)$ también son consideradas útiles. En el lado opuesto, medidas como las normativas no vinculantes de asociaciones profesionales, sindicatos, patronales, etc. $(M=2,79)$ y las normativas no vinculantes del propio medio $(M=2,92)$ fueron consideradas como las menos efectivas. Se puede afirmar que la formación y la concienciación, sobre todo aplicadas a los consumidores, se consideran más efectivas que las normas, sobre todo cuando estas no son de obligado cumplimiento.

Para responder a la PI3.1 continuamos la comparación entre periodismo digital y tradicional, y se aprecia que sólo los dos items que conforman la variable sobre la efectividad de las medidas orientadas a los consumidores muestran diferencias significativas: las campañas de concienciación dirigidas a consumidores se perciben como más significativas por los periodistas online $(M=4,25 ; D T=0,99)$ que por los que trabajan en medios analógicos $(M=3,89 ; D T=1,12), t(380)=$ $-3,258, p<0,01, d=0,33$; igualmente, el desarrollo entre los consumidores de una cultura que rechace prácticas periodísticas irresponsables es considerado más eficaz por los periodistas digitales ( $M=4,46$; $D T=0,87$ ) que por los tradicionales $(M=4,20 ; D T=1,05), t(361,251)=$ $-2,616, p<0,01, d=0,28$.

Aunque su relación es incuestionable, la deontología periodística no se percibe como una herramienta útil para luchar contra las noticias falseadas 


\subsection{Entrevistas}

Los cuatro expertos entrevistados han compartido la mayor parte de resultados de la encuesta, aportando algunos matices. En lo referente a la PI1, no parece haber dudas sobre la gravedad del fenómeno, si bien es cierto que hay una cierta distancia entre las distintas visiones:

- C. R. defiende que este fenómeno es parte de una "guerra híbrida" que "amenaza la democracia y la Unión Europea";

- L. B. resalta los objetivos económicos y políticos que hay detrás de las campañas de noticias falsas que "buscan promover el enfrentamiento";

- J. G. pide "ver las cosas con perspectiva" y no "infravalorar la capacidad crítica de los usuarios", pues "se trata de un fenómeno descontrolado ahora pero que a medio plazo será controlado";

- J. Z. pide no sobredimensionar el fenómeno de la información falsa incluyendo en él las "patochadas" que algunos medios -a menudo, aunque no sólo, digitales- producen para atraer tráfico.

También se apoya la teoría de que las redes sociales son responsables de la dimensión que ha alcanzado el fenómeno: C. R. y L. B. apuntan en esta línea, sobre todo porque, como defiende la segunda, los bulos se difundan con gran rapidez en estas plataformas, que además "embarran el debate". A esta rapidez también aluden J. Z. y J. G., pero matizando el rol de las redes sociales, a las que ven como un canal relevante para su difusión, pero independiente de las fake news. Cabe mencionar también dos nombres propios: C. R. cita el rol de Facebook, mientras J. G. hace lo propio con WhatsApp.

Entrando en la PI2, los expertos consideran que la deontología puede resultar útil, pero no aluden a ella de forma global, sino que se centran en una serie de aspectos: la profesionalidad, la responsabilidad del periodista y, de forma muy notable, la verificación y el contraste de la información. Es relevante en este sentido la afirmación de J. G.:

"Si un periodista difunde una noticia falsa teniendo constancia de que lo es, ya no puede ser periodista".

Finalmente, en lo relativo a la PI3, los cuatro expertos coinciden con lo observado en la encuesta y resaltan la formación de los ciudadanos como una medida efectiva para enfrentarse al fenómeno de la información falseada. La responsabilidad en este proceso de formación recae sobre los propios profesionales y sobre las administraciones públicas, como señalaba C. R., y lo más urgente parece ser la educación en el funcionamiento de la realidad digital. También en línea con lo observado en la encuesta, la formación entre los periodistas es valorada positivamente por los cuatro expertos de cara a luchar contra las noticias falsas, a lo que L. B. añade la legislación como medida efectiva.

\section{Discusión de los resultados}

De acuerdo con los profesionales de la información en España, las causas más relevantes en la aparición de las fake news son las económicas y, en mayor medida, las nuevas formas de comunicar en la Red. Esto concuerda con la investigación previa, que ha analizado la amenaza que la precariedad supone para el periodismo (Figueras-Maz et al., 2012; Bakir; McStay, 2018) y que además ha observado con frecuencia la comunicación online y las redes sociales como causas de las fake news (Marchi, 2012; Allcott; Gentzkow, 2017). En relación con el primer elemento, aunque es necesaria una mayor investigación sobre el tema, la debilidad económica del periodismo hace que los medios tengan mayor tentación de caer en comportamientos como el clickbait -término citado por tres de los expertos- e impide que los periodistas estén blindados frente a las campañas de desinformación orquestadas por grupos de poder, haciendo que se resienta la independencia y aumentando la tentación de defender el discurso interesado -y a menudo falseado- de dichos grupos. Por otro lado es muy destacable la responsabilidad que se otorga a los medios sociales, cuyo modelo de comunicación es central en la época de la posverdad, mas conviene destacar en este campo el rol de quienes comunican sin ser periodistas. Los cuatro expertos consultados indican que la ruptura del monopolio de la comunicación, que ahora pueden ejercer usuarios sin ningún tipo de control, responsabilidad ni conocimiento, es la que en gran parte explica el peso que estas plataformas han tenido en la aparición y proliferación del fenómeno.

La responsabilidad concedida a las nuevas formas de comunicación en internet en la proliferación del fenómeno de las fake news es el único factor en el que se encuentran diferencias destacables al comparar a los periodistas de medios digitales con los de medios tradicionales, ya que los periodistas tradicionales parecen culpar a este factor en mayor medida que quienes trabajan para medios digitales. Ya sea por conocer mejor el funcionamiento del medio o por no asumir los fallos cometidos, realizar periodismo en la Red puede implicar una diferente opinión sobre este asunto, mas la diferencia, aunque significativa, no es grande, pues $d=0,23$ (Cohen, 1988), por lo que será necesario seguir ahondando en este asunto.

Parra-Valero y Oliveira (2018) observaron que la alfabetización informacional era la medida más frecuentemente propuesta por los artículos científicos para enfrentarse a las noticias falsas, algo con lo que concuerdan las opiniones tanto de los periodistas encuestados como de los expertos entrevistados, que destacan no sólo una mayor formación y el desarrollo de una cultura profesional que permitan a los periodistas rechazar prácticas como las noticias falsas, sino también las medidas orientadas a una mayor concienciación y educación de los consumidores. En este último aspecto también se encontraron diferencias significativas, pues los periodistas digitales consideran este tipo de medidas más efectivas que sus colegas de medios tradicionales. De nuevo, esta diferencia puede explicarse por

Junto a la formación y la cultura profesional, las medidas más efectivas contras las noticias falsas parecen ser las orientadas a los consumidores 
un mayor conocimiento del medio o por la no asunción de responsabilidades, motivos que pueden estar detrás de que el conjunto de los profesionales parezca culpar a los consumidores y apunte hacia ellos para combatir las fake news. Esta observación señala la responsabilidad de los consumidores en la aparición del fenómeno de las noticias falsas. Tandoc Jr., Lim y Ling (2017) ya mencionaban el rol de la credibilidad de la audiencia en la construcción de las noticias falseadas, pero es un aspecto en el que todavía no se ha profundizado. Cabe matizarlo en dos sentidos: por un lado, por la posibilidad de que exista un sesgo por el que los periodistas no sean conscientes de su propia responsabilidad o no quieran asumirla; por otro lado, porque su aproximación práctica y conocimiento de la materia, incluyendo las posibles carencias de los consumidores, pueden suponer una perspectiva distinta a la que ofrece la investigación académica.

Un último vacío de conocimiento que esta investigación pretendía llenar guarda relación con la deontología periodística. Se observa que la deontología periodística per se es considerada como una herramienta poco efectiva para enfrentarse a las noticias falsas a pesar de la relación que esta problemática tiene con aspectos centrales de la ética periodística como el contraste de la información. En línea con lo observado por Blanco-Herrero (2018), Díaz-Campo y Segado-Boj (2014; 2015), Micó-Sanz et al. (2008) o Parra-Valcarce, Real-Rodríguez y López-Talavera (2017), se podría esperar que una actualización de las normativas éticas diera lugar a una mejor valoración de éstas como herramientas para luchar contra las noticias falseadas. A su vez, la mayor efectividad que se otorga a las normas obligatorias y la legislación parece chocar con la propia concepción de la deontología, que debe ser autoimpuesta de forma voluntaria, como han indicado Núñez-Encabo (2006) o Real-Rodríguez (2010). Convendría que futuros trabajos ahonden en cómo debe adaptarse la deontología periodística para resultar más útil frente al fenómeno de la información falsa.

\section{Conclusiones}

Este trabajo confirma la investigación previa al señalar las redes sociales y, en general, la comunicación online, así como las causas de índole económica, como principales responsables del surgimiento de las noticias falsas. Al mismo tiempo, tanto la gravedad de este fenómeno como su vinculación con la deontología parecen claras. No obstante, la ética profesional precisa, como han afirmado los autores mencionados en este estudio, de la actualización de sus normativas y de un mayor grado de cumplimiento para poder aumentar su grado de efectividad. Sobre las medidas que resultan más efectivas para combatir las noticias falsas, los encuestados destacan la formación y la concienciación de los consumidores, así como el desarrollo de una cultura profesional entre los periodistas que rechace prácticas irresponsables.

Respondiendo a las preguntas de investigación secundarias, no se han encontrado diferencias importantes entre periodistas digitales y tradicionales en la mayor parte de los contrastes, ni en lo referente a cómo valoran la deontología ni en la forma de aproximarse a las noticias falseadas. No obstante, sí se aprecian algunas diferencias reseñables: la deontología resulta más relevante en medios digitales, al tiempo que los periodistas de estos medios otorgan una menor responsabilidad a los factores relacionados con la comunicación en internet en la aparición de las noticias falsas y consideran más efectivo que los periodistas tradicionales la formación de la audiencia para enfrentar la problemática de las informaciones falsas. El tamaño del efecto de estas diferencias es, en todos los casos, pequeño (Cohen, 1988). Con todo, parece que la aproximación de los periodistas a la deontología y las fake news no varía de forma notable en función del medio en el que trabajan. Todo esto nos permite contribuir a las investigaciones sobre culturas y prácticas profesionales de los periodistas, mostrando más semejanzas que diferencias entre periodistas digitales y tradicionales.

La investigación presentada en este artículo se orienta a periodistas en activo. Sin embargo, dado que entre los miembros de las asociaciones hay periodistas en situación de desempleo, y para no mermar en exceso la muestra, se mantuvo esta categoría, permitiendo al encuestado seleccionar su tipo de periodismo en función de su último empleo. Es posible que esto haya podido causar algún tipo de distorsión en los datos, como también ocurre con periodistas freelance que trabajen para varios tipos de soportes o con profesionales que a lo largo de su trayectoria hayan cambiado de medio, impidiendo una división pura de los tipos de periodismo. Debemos anotar también un posible sesgo en las respuestas, ya que parece improbable que los periodistas indiquen que la deontología no es un elemento relevante en su trabajo o que asuman abiertamente su responsabilidad en la proliferación de las noticias falsas.

En investigaciones futuras se debería seguir indagando en aspectos como la responsabilidad que los periodistas digitales otorgan a la comunicación en internet en la proliferación de las noticias falsas, sobre todo porque la diferencia con respecto a los periodistas tradicionales no es demasiado abultada. También será necesario profundizar en los cambios que la deontología periodística debe llevar a cabo para resultar más efectiva en la lucha contra las noticias falsas. Finalmente, uno de los aspectos que más incógnitas plantea es la responsabilidad que los periodistas otorgan a los consumidores en la proliferación de noticias falsas, pues en la investigación previa apenas se ha prestado atención al receptor, y convendría estudiar las causas que llevan al consumo de este tipo de contenidos. 


\section{Referencias}

Allcott, Hunt; Gentzkow, Matthew (2017). "Social media and fake news in the 2016 election". Journal of economic perspectives, v. 31, n. 2, pp. 211-236.

http://doi.org/10.1257/jep.31.2.211

Alonso-González, Marián (2016). “Información política y fuentes periodísticas en la prensa impresa y en la nativa digital en España”. Communication papers. Media literacy \& gender studies, v. 5, n. 9, pp. 57-74.

https://hdl.handle.net/11441/68532

Alonso, Patricia (2017). “'Fake news' y posverdad en tiempos de populismos: lecciones para periodistas". Cuadernos de periodistas: revista de la Asociación de la Prensa de Madrid, n. 34, pp. 77-84.

http://www.cuadernosdeperiodistas.com/media/2017/07/Patricia_Alonso.pdf

Asociación de la Prensa de Madrid (2015). Informe anual de la profesión periodística 2015. Madrid: Asociación de la Prensa de Madrid.

https://www.apmadrid.es/wp-content/uploads/2016/11/INFORME-PROFESION-APM-2015_baja_7M.pdf

Bakir, Vian; McStay, Andrew (2018). "Fake news and the economy of emotions”. Digital journalism, v. 6, n. 2, pp. 154175.

https://doi.org/10.1080/21670811.2017.1345645

Bennet, W. Lance; Livingston, Steven (2018). "The disinformation order: Disruptive communication and the decline of democratic institutions". European journal of communication, v. 33, n. 2, pp. 122-139.

https://doi.org/10.1177/0267323118760317

Berganza, Rosa; Arcila-Calderón, Carlos; Herrero-Jiménez, Beatriz (2016). “Digital vs. traditional media journalists: Sociodemographic characteristics, and external and internal threats to their autonomy". El profesional de la información, v. 25, n. 2, pp. $179-187$.

https://doi.org/10.3145/epi.2016.mar.04

Blanco-Herrero, David (2018). “Deontología periodística en el nuevo escenario comunicativo: periodismo digital y fake news en los códigos deontológicos del sector en España". En: VI Congreso internacional de la AE-IC. Comunicación y conocimiento. Salamanca 2018, pp. 2812-2828. ISBN: 9788409033935

https://ae-ic.org/final/Libro\%20de\%20Comunicaciones\%20del\%20VI\%20congreso\%20AE-IC.pdf

Carlson, Matt (2018). "Facebook in the news. Social media, journalism, and public responsibility following the 2016 trending topics controversy". Digital journalism, v. 6, n. 1, pp. 4-20.

https://doi.org/10.1080/21670811.2017.1298044

Clark, Chandra; Zhou, Shuhua (2015). "Fake news? A survey on video news releases and their implications on journalistic ethics, independence and credibility of broadcast news". Media watch, v. 6, n. 1, pp. 16-27.

https://doi.org/10.15655/mw/2015/v6i1/55376

Cohen, Jacob (1988). Statistical power analysis for the behavioral sciences. Hillsdale, EUA: Lawrence Erlbaum Associates. ISBN: 0805802835

http://www.utstat.toronto.edu/ brunner/oldclass/378f16/readings/CohenPower.pdf

Colussi-Ribeiro, Juliana (2016). "Ética en la comunidad blogger: una reflexión a partir de la información difundida del atentado terrorista de París en 2015". Razón y palabra, v. 20, n. 2 (93), pp. 497-511.

http://www.revistarazonypalabra.org/index.php/ryp/article/view/32

Comisión Europea (2018). A multi-dimensional approach to disinformation. Report of the independent High level Group on Fake news and Online disinformation. Luxembourg: Publications Office of the European Union.

https://doi.org/10.2759/739290

Cronbach, Lee J. (1951). “Coefficient alpha and the internal structure of tests”. Psychometrika, v. 16, n. 3, pp. $297-334$. https://doi.org/10.1007/BF02310555

Díaz-Campo, Jesús; Segado-Boj, Francisco (2014). "La adaptación de los códigos de ética periodística europeos a internet y las TIC". Ámbitos: Revista internacional de comunicación, n. 26.

http://institucional.us.es/ambitos/?p=1350

Díaz-Campo, Jesús; Segado-Boj, Francisco (2015). "Journalism ethics in a digital environment: How journalistic codes of ethics have been adapted to the Internet and ICTs in countries around the world". Telematics and informatics, v. 32, n. 4, pp. 735-744.

https://doi.org/10.1016/j.tele.2015.03.004

Diezhandino-Nieto, Pilar-María (coord.) (2008). Periodismo en la era de Internet. Claves para entender la situación actual de la información periodística en España. Barcelona: Ariel/Fundación Telefónica. ISBN: 9788408088565 
Elías, Carlos (2018). “Fakenews, poder y periodismo en la era de la posverdad y “hechos alternativos'”. Ámbitos. Revista internacional de comunicación, n. 40.

https://institucionales.us.es/ambitos/?p=3413\#more-3413

Figueras-Maz, Mònica; Mauri-Ríos, Marcel; Alsius-Clavera, Salvador; Salgado-De-Dios, Francesc (2012). "La precariedad te hace dócil. Problemas que afectan a la profesión periodística”. El profesional de la información, v. 21, n. 1, pp. 70-75.

https://doi.org/10.3145/epi.2012.ene.09

Fundéu (2017). “'Noticias falsas' o 'falseadas', mejor que 'fake news'”. Fundéu, 28 septiembre.

https://www.fundeu.es/recomendacion/noticia-falsa-falseada-fake-news

Gómez-Mompart, Josep-Lluís; Gutiérrez-Lozano, Juan-Francisco; Palau-Sampio, Dolors (2015). “La calidad periodística en España según la percepción de los periodistas". Estudios sobre el mensaje periodístico, v. 21, pp. 13-30.

https://doi.org/10.5209/rev_ESMP.2015.v21.50647

Grinberg, Nir; Joseph, Kenneth; Friedland, Lisa; Swire-Thompson, Briony; Lazer, David (2019). "Fake news on Twitter during the 2016 U.S. presidential election". Science, v. 363, n. 6425, pp. 374-378.

https://doi.org/10.1126/science.aau2706

Hayes, Arthur S.; Singer, Jane B.; Ceppos, Jerry (2007). "Shifting roles, enduring values: The credible journalist in a digital age". Journal of mass media ethics, v. 22, n. 4, pp. 262-279.

https://doi.org/10.1080/08900520701583545

Humanes, María-Luisa (1998). "La profesión periodística en España”. Zer. Revista de estudios de comunicación, v. 3, n. 4, pp. 265-278.

http://www.ehu.eus/ojs/index.php/Zer/article/view/17345/15132

Igartua-Perosanz, Juan-José (2006). Métodos cuantitativos de investigación en comunicación. Barcelona: Bosch. ISBN: 9788497902717

Kaplan, Robert M.; Saccuzzo, Dennis P. (1982). Psychological testing. Principles, applications, and issues. Belmont, EUA: Wadsworth. ISBN: 9781133492016

Luengo, María; Maciá-Barber, Carlos; Requejo-Alemán, José-Luis (2017). “Evaluating organisational ethics in Spanish news media". Journalism: Theory, practice \& criticism, v. 18, n. 9, pp. 1142-1162.

https://doi.org/10.1177/1464884916643682

Maciá-Barber, Carlos; Herrera-Damas, Susana (2009). “El acoso mediático a los personajes públicos desde la perspectiva ética de los periodistas madrileños". Revista latina de comunicación social, v. 12, n. 64, pp. 880-893.

https://doi.org/10.4185/RLCS-64-2009-868-880-893

Marchi, Regina (2012). "With Facebook, blogs, and fake news, teens reject journalistic 'objectivity'”. Journal of communication inquiry, v. 36, n. 3, pp. 246-262.

https://doi.org/10.1177/0196859912458700

Marcos-Recio, Juan-Carlos (2017). “Verificar para mejorar la información en los medios de comunicación con fuentes documentales". Hipertext.net, n. 15, pp. 36-45.

https://doi.org/10.2436/20.8050.01.44

Marcos-Recio, Juan-Carlos; García-Alonso, Pedro; Parra-Valcarce, David (2014). "La actividad informativa en los medios digitales: ¿'sobrevivirán los periódicos impresos sin publicidad?”. Estudios sobre el mensaje periodístico, v. 20, n. 1, pp. 177-194. https://doi.org/10.5209/rev_ESMP.2014.v20.n1.45226

Marcos-Recio, Juan-Carlos; Sánchez-Vigil, Juan-Miguel; Olivera-Zaldua, María (2017). “La enorme mentira y la gran verdad de la información en tiempos de la postverdad". Scire, v. 23, n. 2, pp. 13-23.

https://www.ibersid.eu/ojs/index.php/scire/article/view/4446

Martens, Bertin; Aguiar, Luis; Gómez-Herrera, Estrella; Mueller-Langer, Frank (2018). The digital transformation of news media and the rise of disinformation and fake news. Sevilla: European Commission, Joint Research Centre. https://doi.org/10.2139/ssrn.3164170

McNair, Brian (2017). Fake news: Falsehood, fabrication and fantasy in journalism. Londres, Reino Unido: Routledge Focus. ISBN: 9781351392891

McStay, Andrew (2016). "Empathic media and advertising: Industry, policy, legal and citizen perspectives (the case for intimacy)". Big data \& society, v. 3, n. 2, pp. 1-11.

https://doi.org/10.1177/2053951716666868

Micó-Sanz, Josep-Lluís; Canavilhas, João; Masip-Masip, Pere; Ruiz-Caballero, Carles (2008). "La ética en el ejercicio del periodismo: credibilidad y autorregulación en la era del periodismo en internet”. Estudos em comunicação, v. 4, pp. 15-39. 
http://www.ec.ubi.pt/ec/04/html/02-Jose-Lluis-Mico-La-etica-en-el-ejercicio-del-periodismo.html

Núñez-Encabo, Manuel (2006). “Los orígenes del código deontológico de la FAPE”. Cuadernos de periodistas, n. 6, pp. 67-74. http://fape.es/wp-content/uploads/2015/08/Doc2.pdf

Oxford Dictionaries (2016). Word of the year 2016 is...

https://en.oxforddictionaries.com/word-of-the-year/word-of-the-year-2016

Parra-Valcarce, David; Real-Rodríguez, Elena; López-Talavera, María-del-Mar (2017). “Códigos éticos en el ciberperiodismo español y latinoamericano". Naveg@mérica. Revista electrónica editada por la Asociación Española de Americanistas, n. 18, pp. 1-25.

https://revistas.um.es/navegamerica/article/view/286541/208061

Parra-Valero, Pablo; Oliveira, Lídia (2018). "Fake news: una revisión sistemática de la literatura”. (OBS*) Observatorio, v. 12 , n. 5, pp. 54-78.

https://doi.org/10.15847/obsOBS12520181374

Real-Rodríguez, Elena (2010). "La autorregulación. Valoración de los códigos. Conocimiento de los mecanismos de autorregulación”. En: Alsius-Clavera, Salvador; Salgado-De-Dios, Francesc (eds.). La ética informativa vista por los ciudadanos: Contraste de opiniones entre los periodistas y el público, pp. 275-292. Barcelona: Editorial UOC. ISBN: 9788497889383 https://cutt.ly/Lu6OjN

Rodríguez-Martínez, Ruth; López-Meri, Amparo; Merino-Arribas, Adoración; Mauri-Ríos, Marcel (2017). “Instrumentos de rendición de cuentas en España. Análisis comparativo en Cataluña, Galicia, Madrid y Valencia". El profesional de la información, v. 26, n. 2, pp. 255-266.

https://doi.org/10.3145/epi.2017.mar.12

Sánchez-Aranda, José-Javier; Rodríguez-Andrés, Roberto (1999). “Profesionalidad y ética. El caso de los periodistas españoles". Comunicación y sociedad, v. 12, n. 2, pp. 93-114. https://dadun.unav.edu/bitstream/10171/8802/1/20100226105035.pdf

Santín, Marina (2017). "The problem of the right to be forgotten from the perspective of self-regulation in journalism". El profesional de la información, v. 26, n. 2, pp. 303-310.

https://doi.org/10.3145/epi.2017.mar.17

Suárez-Villegas, Juan-Carlos (2015). “Nuevas tecnologías y deontología periodística: comparación entre medios tradicionales y nativos digitales". El profesional de la información, v. 24, n. 4, pp. 390-395.

https://doi.org/10.3145/epi.2015.jul.05

Suárez-Villegas, Juan-Carlos (2017). “El periodismo ciudadano. Análisis de opiniones de periodistas profesionales de España, Italia y Bélgica”. Convergencia. Revista de ciencias sociales, v. 25, n. 74, pp. 91-111.

https://doi.org/10.29101/crcs.v0i74.4383

Suárez-Villegas, Juan-Carlos; Cruz-Álvarez, Jesús (2016). "Los dilemas deontológicos del uso de las redes sociales como fuentes de información. Análisis de la opinión de los periodistas de tres países". Revista latina de comunicación social, v. 71 , pp. 66-84.

https://doi.org/10.4185/RLCS-2016-1084

Suárez-Villegas, Juan-Carlos; Rodríguez-Martínez, Ruth; Mauri-Ríos, Marcel; López-Meri, Amparo (2017). “Accountability y culturas periodísticas en España. Impacto y propuesta de buenas prácticas en los medios de comunicación españoles' (MediaACES)". Revista latina de comunicación social, v. 72, pp. 321-330.

https://doi.org/10.4185/RLCS-2017-1167

Tandoc Jr., Edson C.; Lim, Zheng-Wei; Ling, Richard (2017). “Defining 'fake news'. A typology of scholarly definitions”. Digital journalism, v. 6, n. 2, pp. 137-153.

https://doi.org/10.1080/21670811.2017.1360143

Tirado-Pascual, Nuria (2016). "Corrupción y fuentes informativas en medios tradicionales y nativos digitales". Miguel Hernández communication journal, n. 7, pp. 257-285.

https://dialnet.unirioja.es/servlet/articulo?codigo $=5488474$

Vosoughi, Soroush; Roy, Deb; Aral, Sinan (2018). "The spread of true and false news online”. Science, v. 359, n. 6380, pp. 1146-1151.

https://doi.org/10.1126/science.aap9559

Waisbord, Silvio (2018). "Truth is what happens to news. On journalism, fake news, and post-truth". Journalism studies, v. 19, n. 13 , pp. $1866-1878$.

https://doi.org/10.1080/1461670X.2018.1492881 